IMECE2003-43552

\title{
EMBEDDED ULTRASONICS WITH PIEZOELECTRIC WAFER ACTIVE SENSORS FOR STRUCTURAL HEALTH MONITORING OF THIN-WALL STRUCTURES
}

\author{
Victor Giurgiutiu, PhD, PE, Member ASME \\ Department of Mechanical Engineering \\ University of South Carolina, Columbia, SC 29208 \\ 803.777.8018,victorg@sc.edu
}

\begin{abstract}
The capability of embedded piezoelectric wafer active sensors (PWAS) to perform in-situ ultrasonic nondestructive evaluation (NDE) is explored. Laboratory tests are used to prove that PWAS can satisfactorily perform Lamb wave transmission and reception. Subsequently, pulse-echo method for crack detection in an aircraft panel is illustrated. For large area scanning, a PWAS phased array is used to create the embedded ultrasonics structural radar (EUSR). In conclusion, opportunities for implementation into structural health monitoring applications and further research needs are discussed.
\end{abstract}

\section{INTRODUCTION}

Current structural health monitoring (SHM) research includes: (a) development of embedded active sensors; (b) processing of active sensor data and diagnostics of the health of the structure; (c) prognostics of the remaining structural life. For the health monitoring of an actual structure, networks of embedded active sensors are envisaged. Such sensory networks would be clustered around structural "hot spots". The networks could be wirelessly connected to a data repository and knowledge base.

SHM for requires the development of small, lightweight, inexpensive, unobtrusive, minimally invasive sensors to be embedded in the airframe with minimum weight penalty and at affordable costs [1]. Such sensors should be able to scan the structure and identify the presence of defects and incipient damage.

Current ultrasonic inspection of thin wall structures (e.g., aircraft shells, storage tanks, large pipes, etc.) is a time consuming operation that requires meticulous through-thethickness C-scans over large areas. One method to increase the efficiency of thin-wall structures inspection is to utilize guided waves (e.g., Lamb waves) instead of the conventional pressure waves [2]. Guided waves propagate along the mid-surface of thin-wall plates and shallow shells. They can travel at relatively large distances with very little amplitude loss and offer the advantage of large-area coverage with a minimum of installed sensors [3,4]. Guided Lamb waves have opened new opportunities for cost-effective detection of damage in aircraft structures [5], and a large number of papers have recently been published on this subject [6]. Traditionally, guided waves have been generated by impinging the plate obliquely with a tone-burst from a relatively large ultrasonic transducer. Snell's law ensures mode conversion at the interface, hence a combination of pressure and shear waves are simultaneously generated into the thin plate. However, conventional Lamb-wave transducers (wedge and comb transducers) are relatively too heavy and expensive to be considered for widespread deployment on an aircraft structure as part of a SHM system. Hence, a different type of sensors than the conventional ultrasonic transducers is required for the SHM systems.

Piezoelectric wafer active sensors (PWAS) are inexpensive, non-intrusive, un-obtrusive, and minimally invasive transducers that can be surface-mounted on existing structures, inserted between the layers of lap joints, or put inside composite materials. With PWAS, one can perform embedded ultrasonics, i.e. use the pitch-catch, pulse-echo, and phase array techniques developed for conventional ultrasonics.

PWAS couple their in-plane motion, excited by the applied oscillatory voltage through the piezoelectric effect, with the 
Lamb-waves particle motion on the material surface. Lamb waves can be either quasi-axial $\left(\mathrm{S}_{0}, \mathrm{~S}_{1}, \mathrm{~S}_{2}, \ldots\right)$, or quasiflexural $\left(A_{0}, A_{1}, A_{2}, \ldots\right)$ as shown in Figure 1. PWAS transducers can act as both exciters and sensors of the elastic Lamb waves traveling in the material.

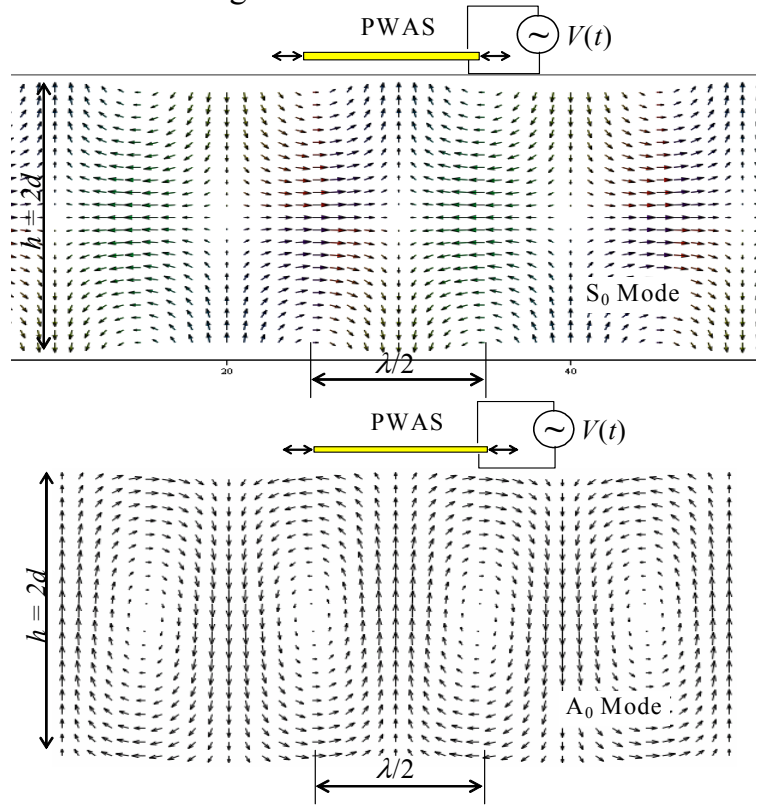

Figure 1 PWAS interaction with $\mathrm{S}_{0}$ and $\mathrm{A}_{0}$ Lamb modes

The PWAS operation is different from the operation of conventional ultrasonic transducers. Not only are PWAS much smaller and cheaper than conventional ultrasonic transducers, but also differ fundamentally in their principles of operation:

(a) PWAS achieve Lamb wave excitation and sensing through surface "pinching" (in-plane strains), while conventional ultrasonic transducers excite through surface "tapping" (normal stress).

(b) PWAS are strongly coupled with the structure and follow the structural dynamics, while conventional ultrasonic transducers are relatively free from the structure and follow their own dynamics.

(c) PWAS are non-resonant wide-band devices, while conventional ultrasonic transducers are narrow-band resonators.

The main advantage of PWAS over conventional ultrasonic transducers lies in their small size, lightweight, low profile, small power, and small cost. In spite of their small size, these novel devices are able to replicate many of the functions of the conventional ultrasonic transducers, as proven by the proof-ofconcept laboratory demonstrations. Pioneering work in this area is credited to Professor Fu-Kuo Chang and his group $[7,8,9]$.

\section{PITCH-CATCH AND PULSE-ECHO WITH PWAS}

The basic principles of Lamb wave transmission and reception with PWAS transducers were verified on simple laboratory experiments performed on a 1.6-mm thick, 2024-aluminum alloy rectangular plate instrumented with eleven 7-mm sq., 0.2mm thick piezoelectric wafers (American Piezo Ceramics Inc., APC-850) placed on a rectangular grid. For pitch-catch experiments, one PWAS (\#11) was as transmitter, while the other ten PWAS were used as receivers. For pulse-echo experiments, the same PWAS (\#11) was used for both transmission and reception. Figure 2a illustrates the pitch-catch method by showing the signals received at PWAS \#1-10 from the transmission at PWAS \#11. Figure 2b illustrates the pulse-echo method, by showing the signal at PWAS \#11, with two distinct regions:

(i) The initial bang, during which the PWAS \#11 acts as transmitter; and

(ii) The echoes zone, containing wave packets R1 through R8 representing echo reflections from the plate boundaries.

These echoes were processed to evaluate the pulse-echo capabilities of the method. Since the wave generated by the initial bang underwent multiple reflections from the plate edges, each of these reflections had a different path length. Loud and clear echoes were recorded from over 2,000 $\mathrm{mm}$ distance, which is remarkable for such small ultrasonic transducers. PWAS are capable of transmitting and receiving pulse-echo signals of remarkable strength and clarity.

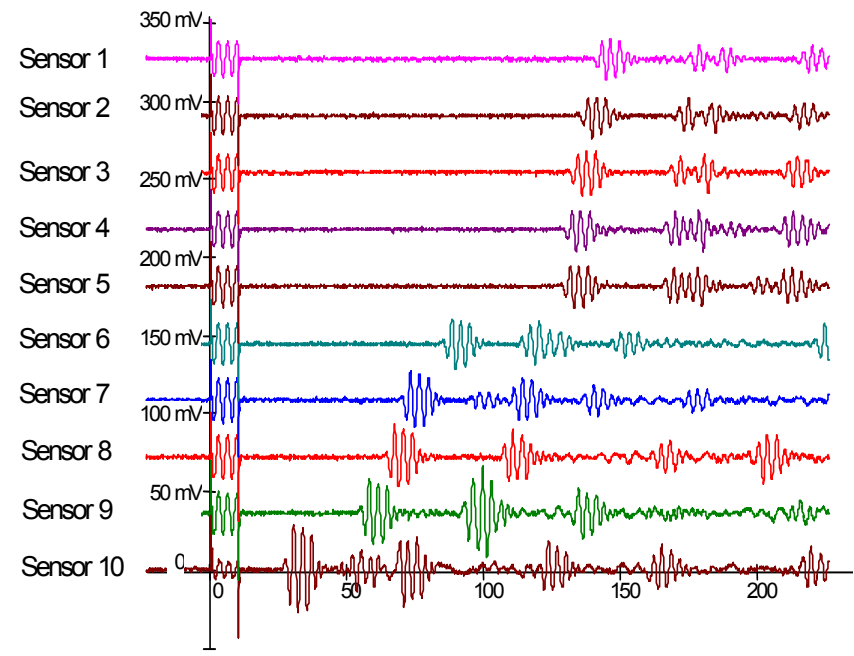

(a)

Time (micro-sec)

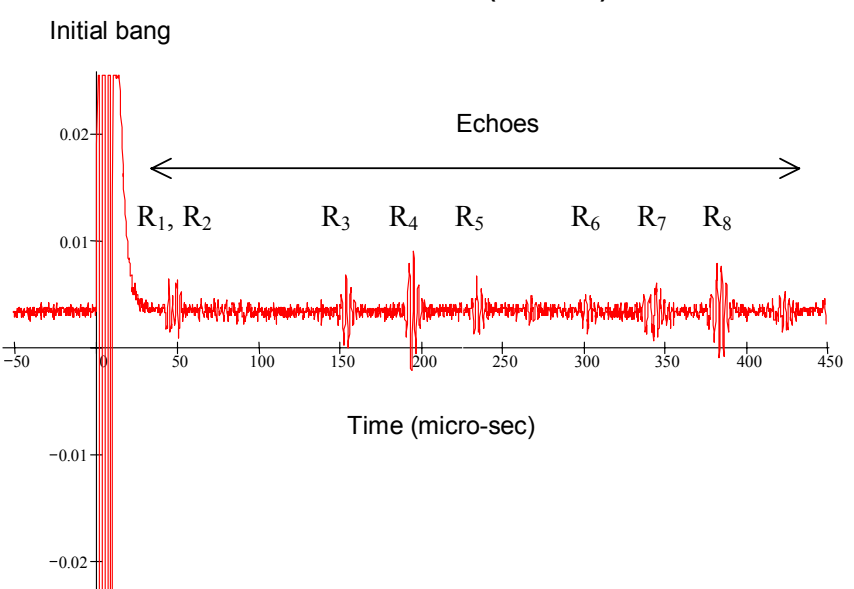

Figure 2 (a) Reception signals on PWAS \#1-10 in pitch catch mode; (b) reflections from plate edges received as echoes at PWAS \#11. 


\section{TUNED LAMB MODE EXCITATION WITH PWAS}

The excitation of Lamb waves in plate with PWAS transducers is studied by considering the excitation applied by the PWAS through a surface stress $\tau=\tau_{0}(x) e^{i \omega t}$. Applying a spacedomain Fourier transform analysis of the basic Lamb wave equations yields the strain wave and displacement wave solutions in the form:

$$
\begin{aligned}
& \varepsilon_{x}(x, t)=\frac{1}{2 \pi} \frac{-i}{2 \mu} \int_{-\infty}^{\infty}\left(\frac{\tilde{\tau} N_{S}}{D_{S}}+\frac{\tilde{\tau} N_{A}}{D_{A}}\right) e^{i(\xi x-\omega t)} d \xi \\
& u_{x}(x, t)=\frac{1}{2 \pi} \frac{-i}{2 \mu} \int_{-\infty}^{\infty} \frac{1}{\xi}\left(\frac{\tilde{\tau} N_{S}}{D_{S}}+\frac{\tilde{\tau} N_{A}}{D_{A}}\right) e^{i(\xi x-\omega t)} d \xi
\end{aligned}
$$

where $\tilde{\tau}$ is the Fourier transform of $\tau_{a}(x), p^{2}=\frac{\omega^{2}}{c_{L}^{2}}-\xi^{2}$, $q^{2}=\frac{\omega^{2}}{c_{T}^{2}}-\xi^{2}$, while $c_{L}^{2}=(\lambda+2 \mu) / \rho, c_{T}^{2}=\mu / \rho$ are the longitudinal (pressure) and transverse (shear) wave speeds, $\lambda$ and $\mu$ are Lame constants, $\rho$ is the mass density, and

$$
\begin{aligned}
& N_{S}=\xi q\left(\xi^{2}+q^{2}\right) \cos p h \cos q h, \\
& D_{S}=\left(\xi^{2}-q^{2}\right)^{2} \cos p h \sin q h+4 \xi^{2} p q \sin p h \cos q h \\
& N_{A}=\xi q\left(\xi^{2}+q^{2}\right) \sin p h \sin q h, \\
& D_{A}=\left(\xi^{2}-q^{2}\right)^{2} \sin p h \cos q h+4 \xi^{2} p q \cos p h \sin q h
\end{aligned}
$$

The expressions $N_{S}, D_{S}, N_{A}, D_{A}$ are related to the RayleighLamb equations for symmetric and antisymmetric Lamb modes. Note that $p$ and $q$ depend on $\xi$, thus increasing the complexity of the problem. The integral in Equation (2) is singular at the roots of $D_{S}$ and $D_{A}$. The equations $D_{S}=0$ and $D_{A}$ $=0$ are exactly the Rayleigh-Lamb characteristic equations for symmetric and anti-symmetric motions accepting the simple roots:

$$
\xi_{0}^{S}, \xi_{1}^{S}, \xi_{2}^{S}, \ldots \quad \xi_{0}^{A}, \xi_{1}^{A}, \xi_{2}^{A}, \ldots
$$

corresponding to the symmetric (S) and anti-symmetric (A) Lamb waveguide modes. The evaluation of the integral in Equation (2) is done by the residue theorem, using a contour consisting of a semicircle in the upper half of the complex $\xi$ plane and the real axis.

For ideal bonding between the PWAS and the plate, the shear stress in the bonding layer and the corresponding spacedomain Fourier transform are:

$\tau(x)=a \tau_{0}[\delta(x-a)-\delta(x+a)], \quad \tilde{\tau}=a \tau_{0}[-2 i \sin \xi a]$

Hence, the strain-wave solution becomes:

$$
\begin{aligned}
\varepsilon_{x}(x, t) & =-i \frac{a \tau_{0}}{\mu} \sum_{\xi^{S}} \sin \xi^{S} a \frac{N_{S}\left(\xi^{S}\right)}{D_{S}^{\prime}\left(\xi^{S}\right)} e^{i\left(\xi^{S} x-\omega t\right)} \\
& -i \frac{a \tau_{0}}{\mu} \sum_{\xi^{A}} \sin \xi^{A} a \frac{N_{A}\left(\xi^{A}\right)}{D_{A}^{\prime}\left(\xi^{A}\right)} e^{i\left(\xi^{A} x-\omega t\right)}
\end{aligned}
$$

Similarly, the displacement wave solution is obtained as:

$$
\begin{aligned}
u_{x}(x, t) & =-i \frac{a \tau_{0}}{\mu} \sum_{\xi^{S}} \frac{\sin \xi^{S} a}{\xi^{S}} \frac{N_{S}\left(\xi^{S}\right)}{D_{S}^{\prime}\left(\xi^{S}\right)} e^{i\left(\xi^{S} x-\omega t\right)} \\
& -i \frac{a \tau_{0}}{\mu} \sum_{\xi^{A}} \frac{\sin \xi^{A} a}{\xi^{A}} \frac{N_{A}\left(\xi^{A}\right)}{D_{A}^{\prime}\left(\xi^{A}\right)} e^{i\left(\xi^{A} x-\omega t\right)}
\end{aligned}
$$

A plot of these equations up to $1000 \mathrm{kHz}$ is presented in Figure 3 . Equations (5) and (6) contains the $\sin \xi a$ behavior that displays maxima when the PWAS length $l_{a}=2 a$ equals an odd multiple of the half wavelength, and minima when it equals an even multiple of the half wavelength. A complex pattern of such maxima and minima emerges, since several Lamb modes, each with its own different wavelength, coexist at the same time. Figure 3 shows the remarkable fact that, at $300 \mathrm{kHz}$, the amplitude of the $\mathrm{A}_{0}$ mode goes through zero, while that of the $\mathrm{S}_{0}$ is close to its peak. This represents an excitation "sweet spot" for $S_{0}$ Lamb waves. Experimental results confirming this prediction are presented in Figure $3 b$.

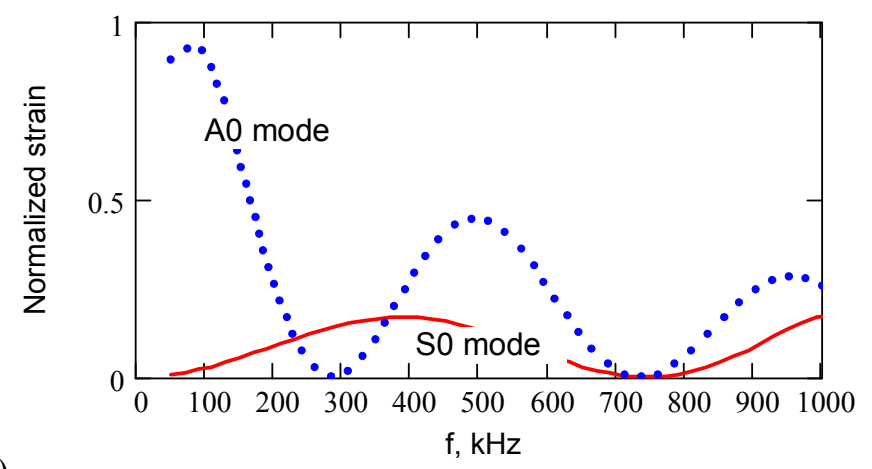

(a)

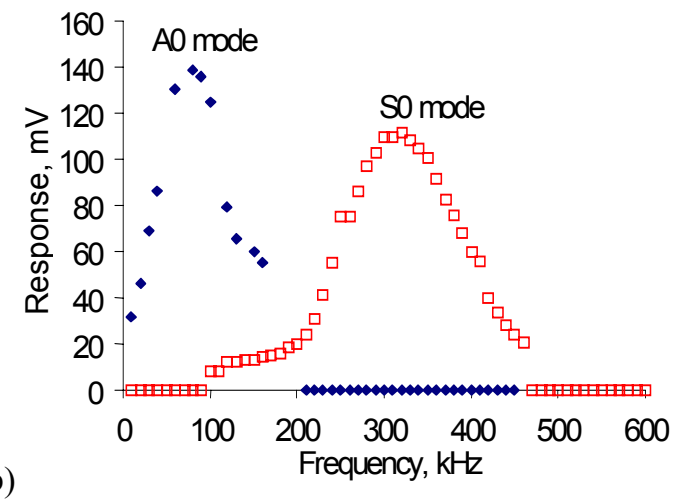

Figure 3 (a) Strain Lamb wave amplitudes predicted by the Lamb wave PWAS excitation tuning $\left(\mathrm{S}_{0}=\right.$ continuous line; $\mathrm{A}_{0}=$ dotted line $)$; (b) excitation sweet spot observed experimentally at $300 \mathrm{kHz}$ in a 1.6-mm aluminum plate under a 7-mm PWAS excitation

This proves that frequencies can be found for which the response is dominated by certain preferentially excited modes. This is wavelength-based mode tuning. Another factor that must be considered in Lamb wave tuning under PWAS excitation is the mode amplitude at the plate surface. This factor is contained in the values taken for each mode by the function $N / D^{\prime}$. Hence, it is conceivable that, at a given frequency, some higher modes may have less surface amplitudes, while other may have larger surface amplitudes. Thus, two important factors for the design of PWAS- 
based Lamb-wave embedded NDE for structural health monitoring have been identified:

a) The variation of $|\sin \xi a|$ with frequency for each Lamb wave mode

b) The variation of the surface strain with frequency for each Lamb wave mode

Equations (6) and (7) can be viewed as a superposition of waveguide Lamb modes. In this case, the contribution of each mode in these equations can be expressed in the general form [6]:

$$
A_{\xi}(x, t)=G \cdot E_{\xi} \cdot F \cdot e^{i(\xi x-\omega t)}
$$

where $G$ is a numerical factor representing the PWAS transducer power transmitted to the structure, $E_{\xi}$ is the excitability function of mode $\xi$, and $F$ is the Fourier transform of the excitation.

Lamb wave mode tuning is important because various Lamb wave modes are sensitive to various types of damage. Our research has shown that $\mathrm{S}_{0}$ Lamb mode is more sensitive to through the thickness cracks. The $\mathrm{S}_{0}$ mode's reflection from a through the thickness crack is several times stronger than that of the $\mathrm{A}_{0}$ mode. On the other hand, the $\mathrm{A}_{0}$ mode is more sensitive to corrosion and delamination damage.

\section{PWAS PITCH-CATCH FOR DISBOND DETECTION}

The PWAS pitch-catch method was used for detecting disbonds in adhesive joint. Figure 4 shows the instrumentation setup used in this investigation. Two specimens were used. Each specimen consisted of two $178 \mathrm{~mm}$ x $36 \mathrm{~mm}$ x $1.6 \mathrm{~mm} 2024$ aluminum strips bonded together with cold-cured Loctite Hysol EA 9309.3NA epoxy adhesive system. One specimen was designated pristine. The other specimen was designated with disbond. In this latter specimen, a $25 \mathrm{~mm}$ wide simulated disbond was created in the middle during the bonding process, using a $25 \mu \mathrm{m}$ (0.001 in) Mylar strip. The experimentation was done with a $1,200 \mathrm{kHz}$ smoothed tone burst signal that was transmitted from PWAS \#1 and received at PWAS \#3a and
PWAS \#3b, which are located on the top and the bottom of the specimen. The received signals were captured on a digital oscilloscope and then stored in a laptop PC. During the experiments, it was observed that the presence of the disbond clearly modifies the signal character.

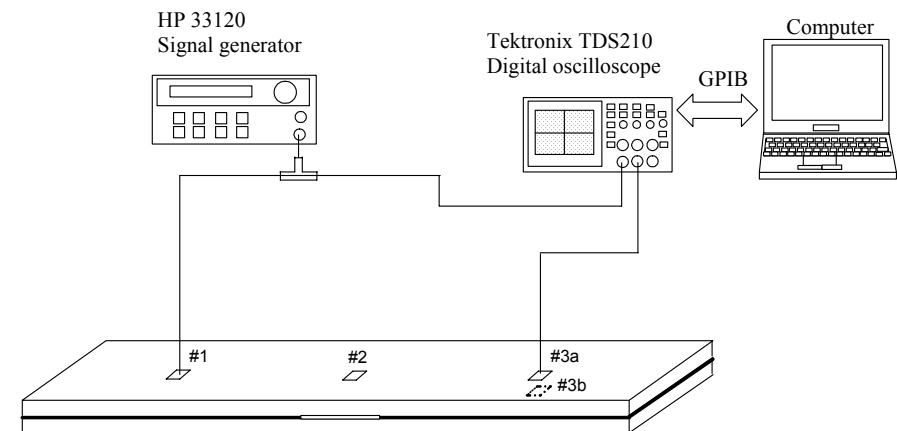

Figure 4 Pitch-catch setup for disbond detection with PWAS Instrumentation setup used for pitch-catch method to send and receive Lamb waves from one sensor to another one

Figure 5 presents a plot of the results. Four signals are displayed. Display shift was used to separate the signals graphically. The upper two signals represent the signals received at the top and bottom PWAS in the pristine specimen. It is apparent that the two signals are more or less identical. The lower two signals are the signals received at the top and bottom of the specimen with disbond. It is apparent that a clear difference exists between the top and the bottom signals. In addition, these signals are clearly different from the signals in the pristine specimen. The time of flight of the signal from the bottom PWAS is clearly longer than that of the signal from the top PWAS. We do not have yet a complete explanation, of this phenomenon, which needs more detailed examination, backed up by theoretical analysis. However, it is clear that the pitch-catch method can be successfully used for disbond detection using Lamb waves transmitted and received with embedded PWAS.

\section{Top PWAS}

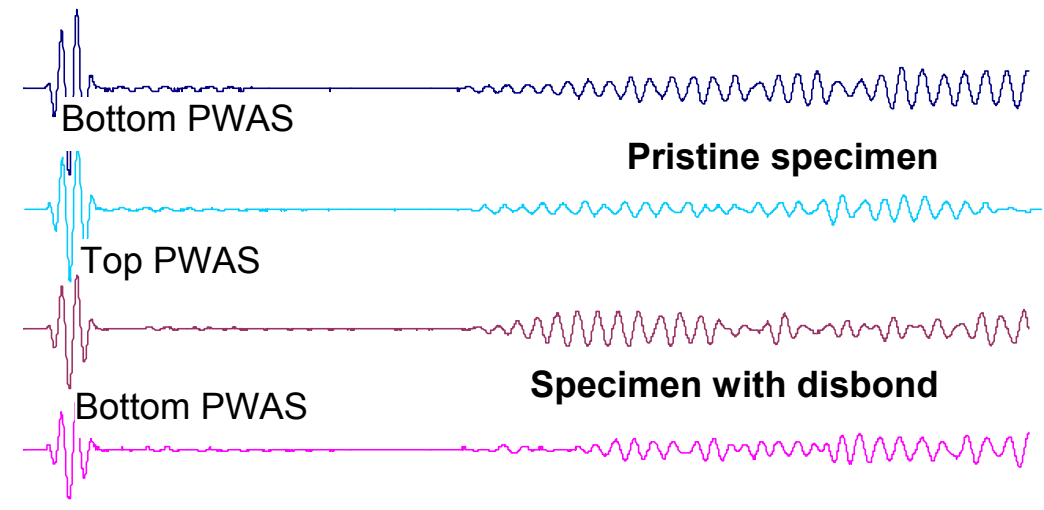

\begin{tabular}{ccccc}
\hline 0.00 & 10.00 & 20.00 & 30.00 & 40.00
\end{tabular}

Figure 5 Lamb wave signals transmitted from top PWAS \#1 and received at top PWAS (\#3a) and bottom PWAS (\#3b), in the pristine and disbond cases. 


\section{PWAS PULSE-ECHO FOR CRACK DETECTION}

Wave propagation experiments were conducted on an aircraft panel to illustrate crack detection through the pulseecho method. The panel has a typical aircraft construction, featuring a vertical splice joint and horizontal stiffeners. Figure 6 shows three photographs of PWAS installation on increasingly more complex structural regions of the panel. Adjacent to the photographs are the PWAS signals. All the experiments used only one PWAS, operated in pulse-echo mode. The PWAS was placed in the same relative location, i.e., at $200 \mathrm{~mm}$ to the right of the vertical row of rivets. The first row of Figure 6 shows the situation with the lowest complexity, in which only the vertical row of rivets is present in the far left. The signal to the right of this photograph shows the initial bang (centered at around 5.3 micro-sec) and multiple reflections from the panel edges and the splice joint. The echoes start to arrive at approximately $60 \mu \mathrm{s}$. The second row of Figure 6 shows the vertical row of rivets in the far left and, in addition, a horizontal double row of rivets stretching towards the PWAS. The signal to the right shows that, in addition to the multiple echoes from the panel edges and the splice, the PWAS also receives diffraction backscatter echoes from the rivets located at the beginning of the horizontal row.

These backscatter echoes are visible at around $42 \mu \mathrm{s}$. The third row in Figure 6 shows a region of the panel similar to that presented in the previous row, but having an additional feature: a simulated crack (12.7-mm EDM hairline slit) emanating from the first rivet hole in the top horizontal row. The signal at the right of this photo shows features similar to those of the previous signal, but somehow stronger at the $42 \mu$ s position. The features at $42 \mu$ s correspond to the superposed reflections from the rivets and from the crack. The detection of the crack seems particularly difficult because the echoes from the crack and from the rivets are superposed. This difficulty was resolved by the differential signal method, i.e., subtracting the signal presented in the second row from the signal presented in the third row.

In practice, such a situation would correspond to subtracting a signal previously recorded on the undamaged structure from the signal recorded now on the damaged structure. Such a situation of using archived signals is typical of health monitoring systems. When the two signals were subtracted, the result presented in the last row of Figure 6 was obtained. This differential signal shows a "loud and clear" echo due entirely to the crack. The echo, marked "reflection from the crack" is centered at $42 \mu$ s, i.e., TOF $=37 \mu$ s which correlates very well with a $5.4 \mathrm{~km} / \mathrm{s} 200-\mathrm{mm}$ total travel from the PWAS to the crack placed at $100 \mathrm{~mm}$. The cleanness of the crackdetection feature and the quietness of the signal ahead of the crack-detection feature are remarkable. Thus, we concluded that PWAS are capable of clean and un-ambiguous detection of structural cracks.

\section{PWAS PHASED ARRAYS FOR CRACK DETECTION}

The advantages of phased array transducers for ultrasonic testing are multiple $[10,11]$. Krautkramer, Inc. produces a line of phased array transducers for the inspection of very thick specimens, and in the sidewise inspection of thick slabs, etc. These transducers employ pressure waves generated through normal impingement on the material surface.

In our research [12], we developed a phased array technology for thin wall structures (e.g., aircraft shells, storage tanks, large pipes, etc.) that uses Lamb waves to cover a large surface area through beam steering from a central location. We called this concept embedded ultrasonics structural radar (EUSR) and constructed a simple proof-of-concept experiment (Figure 7a). A PWAS array was made up of a number of identical 7-mm sq. elements aligned at uniform 9-mm pitch. The PWAS phased array was placed at the center of a $4-\mathrm{ft}$ square thin aluminum plate (Figure 7a). The wave pattern generated by the phased array is the result of the superposition of the waves generated by each individual element. By sequentially firing the individual elements of an array transducer at slightly different times, the ultrasonic wave front can be focused or steered in a specific direction. Thus, we achieved electronic sweeping and/or refocusing of the beam without physical manipulating the transducers. We proved that inspection of a wide zone is possible by creating a sweeping beam of ultrasonic Lamb waves that covered the whole plate. Once the beam steering and focusing was established, the detection of crack was done with the pulse-echo method.

During these proof-of-concept experiments, the EUSR methodology was used to detect cracks in two typical situations: (i) a $19-\mathrm{mm}$ broadside crack placed at $305 \mathrm{~mm}$ from the array in the 90 deg direction; and (ii) a $19-\mathrm{mm}$ broadside crack placed at $409 \mathrm{~mm}$ from the array in the $136 \mathrm{deg}$ direction. Of these two, the latter was more challenging because the ultrasonic beam is not reflected back to the source but rather deflected sideways. Hence, the echo received from the offside crack is merely the backscatter signal generated at the crack tips. Figure $7 \mathrm{~b}$ presents the front panel GUI of the embedded ultrasonic structural radar graphical user interface (EUSR-GUI) displaying the offside signals. The sweep is performed automatically to produce the structural defect image in the right pane. Manual sweep can be performed with the turn knob. The reconstructed signal is shown in the lower pane. In Figure $7 b$, the lower pane show the signal reconstructed at the beam angle $\phi_{0}=136 \mathrm{deg}$ corresponding to the crack location.

\section{PWAS OPPORTUNITIES FOR VEHICLE STRUCTURAL HEALTH MONITORING}

PWAS are a key component being examined for identifying incipient flaws, principally corrosion and cracking, in the complex geometric structure of aircraft, or other thin wall structures. The significant research is focused on early crack detection in structural joints. Most effort to date has been directed at increasing the sensitivity of existing inspection tools and developing rapid scanning platforms that allow fast automated scanning of large areas on the aircraft. 

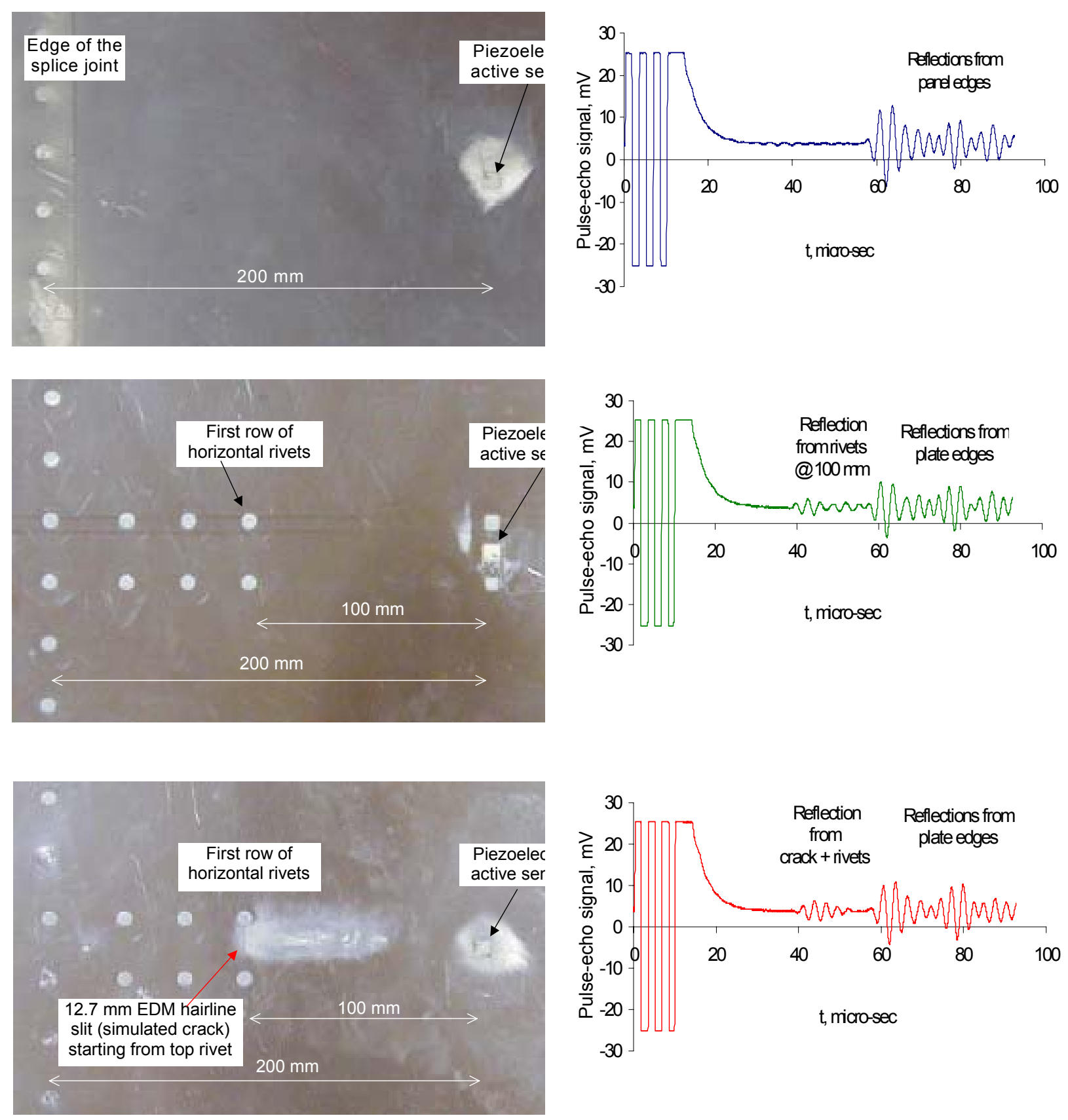

Figure 6 Crack-detection laboratory experiments on an aircraft panel: left column represents specimens (40-mil 2025 T3) with increasing complexity. Right column represents the pulse-echo signals. Fourth cell in the right column shows the crack detection through the differential signal method.

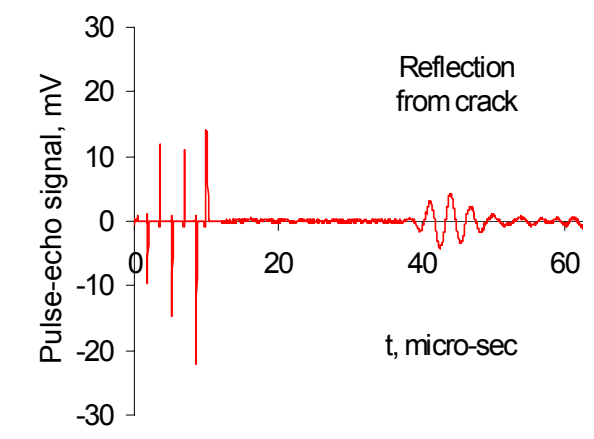




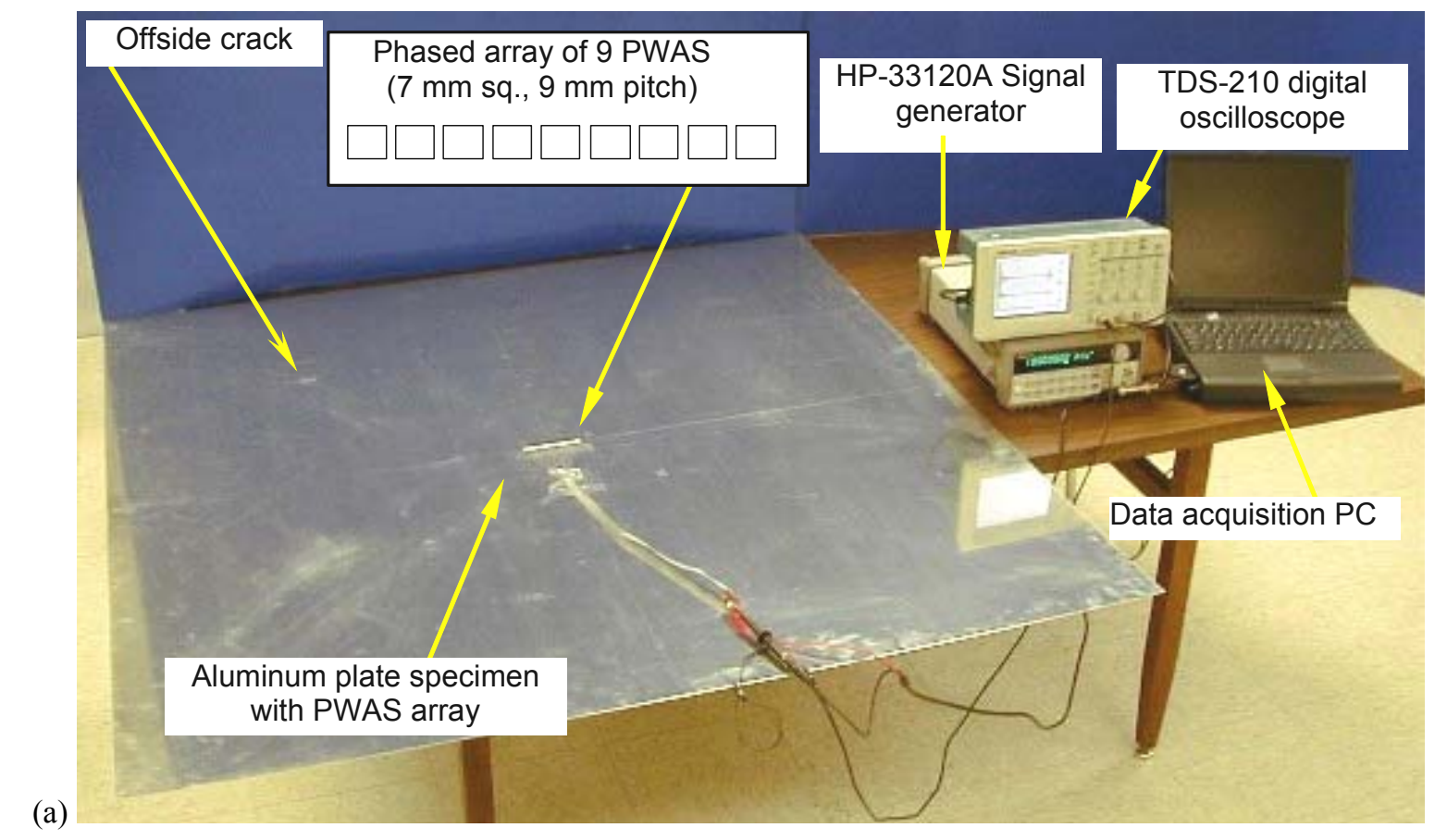

(a)

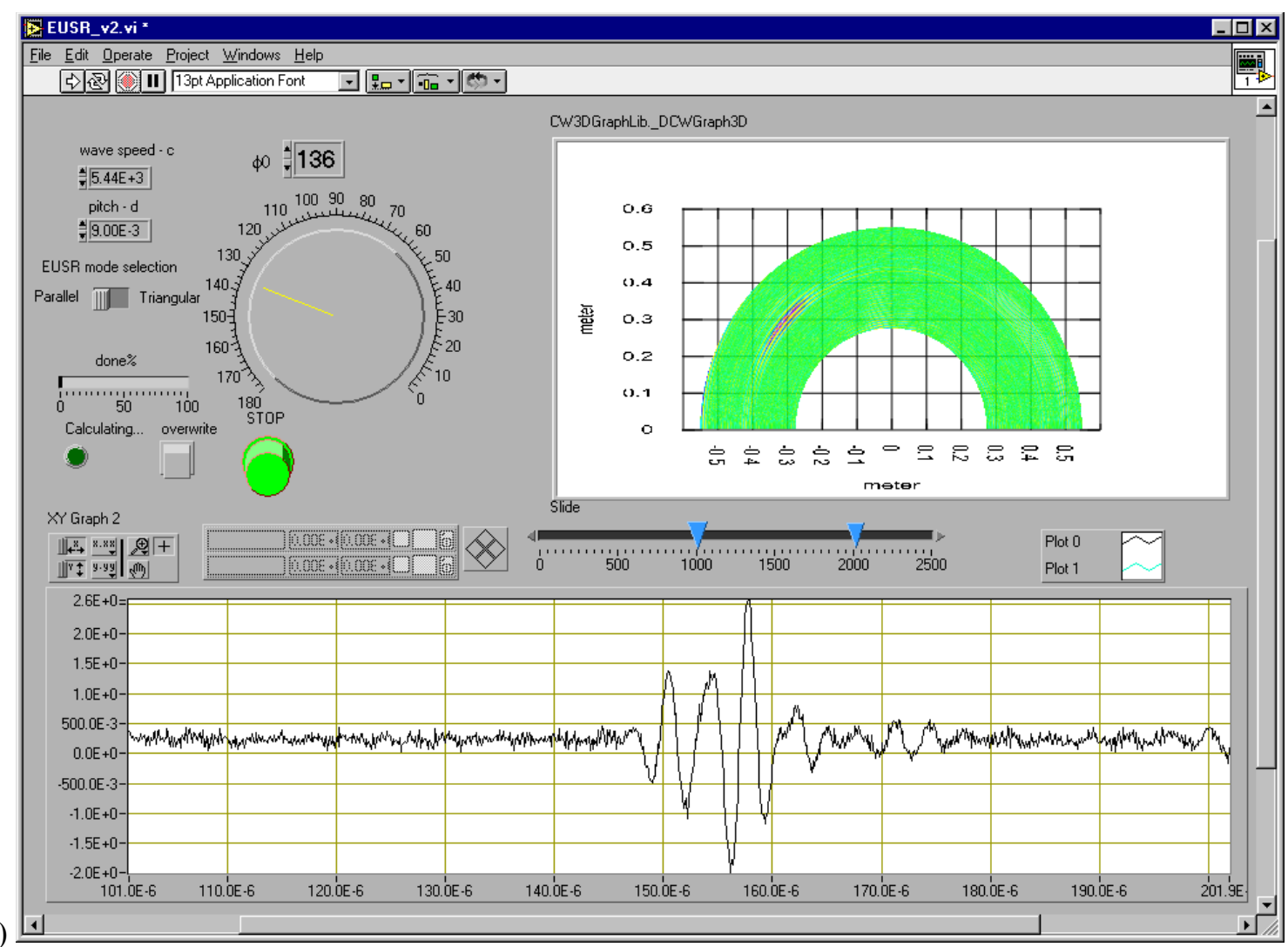

Figure 7 - Proof-of-concept EUSR experiment: (a) thin plate specimen 9-element PWAS array and 19-mm offside crack; (b) Graphical user interface (EUSR-GUI) front panel. The angle sweep is performed automatically to produce the structure/defect imaging picture on the right. Manual sweep of the beam angle can be also performed with the turn knob; the signal reconstructed at the particular beam angle (here, $\phi_{0}=136 \mathrm{deg}$ ) is shown in the lower picture. 
Since significant improvements have already been made in this area, the next frontier to address in this area is the use of on-board health monitoring.

Structural health monitoring that provides owners and maintainers of long life expectancy vehicles is being envisioned to localize potential problem areas in the acreage of aircraft skin and underlying structure. This knowledge of evolving "hot spots" will then be used to target inspection techniques that will allow detailed characterization of the structure, which can be used to determine the appropriate maintenance and/or repair needs.

This health monitoring approach has the advantage of a long-term change in maintenance philosophy. The current scheduled maintenance approach that relies on a "find and fix" process will evolve into a demand driven "fix when required" approach.

One conceptual vision for a structural health monitoring system is that of an artificial central nervous system. PWAS are seen as leading candidates to serve as one specialized type of "neuron" in this systems level concept.

\section{RESEARCH NEEDS IN PWAS-BASED STRUCTURAL HEALTH MONITORING AND EMBEDDED NDE}

Many aspects of the use and usability of PWAS still need to be investigated. In a long-term perspective, the entire range of aircraft structure will eventually be instrumented with sensors and a key goal for PWAS is to determine where and how they will perform most effectively. Early investigations are focused on aircraft skin and lap joints. A majority of existing aircraft have $1 \mathrm{~mm}(0.040$ ") to $1.6 \mathrm{~mm}$ $(0.065 ")$ thin skin made of 7075-T6 and 2024 aluminum alloys, though many other alloys less common and some very thick skin exists in certain locations. Lap joint configurations vary widely from standard two layer configuration (with or without sealant) towards much more complex joints with multiple layers that may include one or more doublers. Rivets, screws and fasteners of may types are used to bind these lap joints and many joints contain attachment to various structural components such as ribs, spars, bulkheads, etc. The growth in the use of organic composites in aircraft structure introduces a whole new set of variables for health monitoring. Determining how PWAS can be used in these structures to detect fatigue cracking, corrosion growth (particularly in the advent of $>10 \%$ material loss), and stress corrosion cracking is the first step in this large area of research. It seems intuitive that PWAS will not be the optimal sensing tool for every case of structural monitoring: an understanding of which facet of this problem is suited for PWAS is required.

Another key aspect of structural health monitoring involves sensors that will have a very long useful life in service. It is anticipated that PWAS will be installed into an aircraft during depot maintenance activities when much of the skin is removed for inspection and repair. The typical time between depot maintenance can run from 6 to 8 years, so sensor durability and survivability are critical. Air turbulence and buffet will cause significant vibration and repetitive stress to the PWAS. Temperature swings from lows of $-40^{\circ} \mathrm{F}$ to highs of $150^{\circ} \mathrm{F}$ are possible. Corrosive effects of salts, de-icing agents, and many types fuels, fluids and lubricants are also present. Clearly, surviving in an operational environment is no small challenge.

Lastly, the usability of these sensors and their associated support systems, including power, network connectivity, and control systems must be integrated into the operational aircraft. Once all these hurdles have been addressed, PWAS will be ready for implementation into the Air Force's Fleet.

\section{CONCLUSIONS}

A novel structural health-monitoring concept embedded ultrasonics with piezoelectric wafer active sensors (PWAS) - has been presented. PWAS can be structurally embedded as both individual transducers and phased arrays. They can be placed even inside closed cavities during fabrication/overhaul (such as wing structures), and then be left in place for the life of the structure. The embedded ultrasonics concept opens new horizons for performing in-situ damage detection and structural health monitoring of a multitude of thin-wall structures.

Proof-of-concept experiments have been presented that verified that "loud and clear" ultrasonic Lamb wave signals can be successfully generated and detected with the small, inexpensive, unobtrusive, low-power and lowcost PWAS transducers. The omnidirectional transmission of Lamb waves, and the relatively long distance propagation (more than $2000 \mathrm{~mm}$ ) were proven. The pitch-catch method was used to perform disbond detection in an adhesive joint. The pulse-echo crackdetection method was demonstrated on a realistic aircraft panel. PWAS phased arrays were constructed to perform ultrasonic scanning beam experiments for crack detection in large areas. Successful detection of broadside and offside cracks in a large plate was illustrated with a simple experimental setup and a comprehensive signal processing and graphical user interface (EUSR-GUI).

This multi-use capability of PWAS-based embedded ultrasonics opens important opportunities for their use in 
structural health monitoring, damage detection, and failure prevention of thin wall structures. However, this emerging technology requires a sustained R\&D effort to achieve its full developmental potential.

\section{ACKNOWLEDGMENT}

2002 Summer Faculty Fellowship Program at the Air Force Research Laboratory NDE Branch, WrightPatterson AFB, OH

\section{REFERENCES}

1. Kropas-Hughes, C.V.; Perez, I.; Winfree; W. P.; Motzer, W. P.; Thompson, R. B. (2002) "Vision of Future Directions of NDE Research" in Review of Quantitative Nondestructive Evaluation Vol. 21, ed. by D. O. Thompson and D. E. Chimenti, American Institute of Physics, Vol. 615, 2002, pp. 2042-2051

2. Rose, J. L. (2002) "A Baseline and Vision of Ultrasonic Guided Wave Inspection Potential", ASME Journal of Pressure Vessel Technology Special Issue on Nondestructive Characterization of Structural Materials, Vol. 124, No. 3, August 2002, pp. 273-282

3. Viktorov, I. A. (1967) Rayleigh and Lamb Waves, Plenum Press, New York, 1967

4. Rose, J. L. (1999) Ultrasonic Waves in Solid Media, Cambridge University Press, 1999

5. Dalton, R. P.; Cawley, P.; Lowe, M. J. S. (2001) "The Potential of Guided Waves for Monitoring Large Areas of Metallic Aircraft Structure", Journal of Nondestructive Evaluation, Vol. 20, pp. 29-46, 2001

6. Thomson, D. O. and Chimenti, D. E. (Editors) (2002) Review of Progress in Quantitative Nondestructive Evaluation, Chapter 2C "Guided Waves" and Chapter 7 "NDE Applications", AIP Conference Proceedings Vol. 615, 2002

7. Chang, F.-K. (1995) "Built-In Damage Diagnostics for Composite Structures", in Proceedings of the $10^{\text {th }}$ International Conference on Composite Structures (ICCM-10), Vol. 5, Whistler, B. C., Canada, August 14-18, 1995, pp.283-289

8. Chang, F.-K. (1998) "Manufacturing and Design of Built-in Diagnostics for Composite Structures", $52^{\text {nd }}$ Meeting of the Society for Machinery Failure Prevention Technology, Virginia Beach, VA, March 30 - April 3, 1998.

9. Chang, F.-K. (2001) "Structural Health Monitoring: Aerospace Assessment", Aero Mat 2001, 12th ASM Annual Advanced Aerospace Materials and Processes Conference, 12-13 June 2001, Long Beach CA.

10. Krautkramer, J.; Krautkramer, H. (1990) Ultrasonic Testing of Materials, Springer-Verlag, 1990

11. Lines D.; Dickson K. (1999) "Optimization of HighFrequency Array Technology for Lap-Joint Inspection"; Proceedings of the 3rd Joint Conference on Aging Aircraft, 1999, www.galaxyscientific.com/agingaircraft/index2.htm

12. Giurgiutiu, V.; Bao, J. (2002) "Embedded Ultrasonic Structural Radar for Nondestructive Evaluation of Thin-Wall Structures" Proceedings of the 2002 ASME International Mechanical Engineering Congress, November 17-22, 2002, New Orleans, LA, paper \# IMECE2002-39017 